\title{
DER DANZÓN UND DESSEN FORTWIRKEN IN AUSGEW ̈̈HLTEN INTERPRETATIONEN VON Gonzalo RuBALCABA
}

\author{
Christa Bruckner-Haring
}

\section{Einleitung}

Der Danzón nimmt eine wichtige Position sowohl in der kubanischen Musikgeschichte als auch in der frühen Entwicklung des Jazz ein. Das in Kuba aus europäischen Vorformen entwickelte Genre wurde binnen kurzer Zeit zum beliebtesten und repräsentativen Nationaltanz Kubas und war die Basis für weitere in Kuba entstandene Musikstile, wie den Danzón de Nuevo Ritmo, auch bekannt als Mambo, oder den Cha-Cha-Cha. Zudem hatte der Danzón einen großen Einfluss auf die frühen Formen des Jazz, einerseits durch die Einbindung der kubanischen Rhythmuskonzepte und Rhythmusformeln - die so genannten Claves - , andererseits insbesondere durch die Bedeutung der typischen Instrumental-Formationen der Danzones und deren Spielweisen für die Stilistik der Dixieland-Ensembles und deren Bläsersätze. Laut Díaz Ayala (2011) werden diesbezügliche Einflüsse beispielsweise im Vergleich der Aufnahmen der Ensembles Orquesta de Felipe Valdés und Original Dixieland Jazz Band deutlich. Die geographische Nähe der USA und Kubas brachte eine häufige Interaktion von Musikerinnen und Musikern mit sich, wodurch sich wechselseitige Beeinflussungen von Jazz und kubanischer Musik ergaben. Frühe Beispiele für nordamerikanische Jazzeinflüsse in kubanischer Musik sind unter anderem bei den Ensembles Orquesta Arcaño, Los Jardineros und Rico's Creole Band zu finden (vgl. ebd.).

Die althergebrachte Offenheit der kubanischen Musikstile für fremde Einflüsse setzte sich auch in den Stilistiken des kubanischen Jazz fort. Inzwischen als eigenständiger Bereich des so genannten »Latin Jazz« betrachtet, werden im »Jazz Cubano « oder »Cuban Jazz« bis in die Gegenwart herauf Verbindungen kreiert zwischen traditioneller kubanischer Musik und Jazz 
wie auch zwischen dem traditionellen einheimischen Repertoire und diversen Jazz-Stilistiken. Diese Musizierpraxis hat eine mittlerweile langjährige Tradition, und ihr gehört eine Vielzahl kubanischer Musikerinnen und Musiker an. Eine besondere Rolle spielt in Kuba hierbei das Vorhandensein von Musikerfamilien, die sich speziell der Pflege traditioneller Stile widmen. Dieser Tradition entstammen bedeutende kubanische Jazzpianisten wie z.B. Bebo Valdés (*1918) und Chucho Valdés (*1941), Guillermo Rubalcaba (*1927) und Gonzalo Rubalcaba (*1963), Ernán López Nussa (*1958) und Harold López Nussa (*1983) (vgl. Gómez Sotolongo 2011).

Gonzalo Rubalcaba gilt als herausragender Vertreter des zeitgenössischen Jazz Cubano und zudem als jemand, der besonders stark mit kubanischen Traditionen verbunden ist. Hineingeboren in eine Musikerfamilie, wuchs Rubalcaba inmitten einer intensiv gepflegten Musiktradition auf, wobei sich seine Familie hauptsächlich dem Erbe des Danzón widmete. Rubalcaba tradiert dieses Erbe weiter, indem er musikalische Charakteristika und Konzepte des Danzón in seinen Interpretationen und Eigenkompositionen verwendet. In der vorliegenden Untersuchung wird mit »El Cadete Constitucional« ein ausgewähltes Stück aus der Tradition des Danzón besprochen, und es werden Rubalcabas Interpretationen eben dieses Stückes analysiert.

\section{Der Danzón und »El Cadete Constitucional«}

Der Danzón ist ein Musikstil, der sich aus den populären europäischen Country Dances des 17. und 18. Jahrhunderts entwickelte: Der aus England bekannte und beliebte Country Dance gelangte rasch auf das europäischen Festland (Deutschland: Kontratanz; Frankreich: Contredanse; Spanien: Contradanza) und im späten 18. Jahrhundert mit europäischen Emigranten auch nach Kuba. Aus der Contradanza entwickelte sich während des 19. Jahrhunderts die Danza (dt. »Tanz«) und aus dieser wiederum der Danzón, was übersetzt »großer Tanz« bedeutet. Der erste offiziell als solcher vorgestellte Danzón ist »Las Alturas De Simpsón«, komponiert von Miguel Faílde Pérez (1852-1921), uraufgeführt am Neujahrstag 1879 in Matanzas (vgl. Giro 2007: 8ff.).

Gespielt wurden Danzones anfänglich von einem Orquesta Típica (dt. »Typisches Orchester«), eine Formation bestehend aus Holz- und Blechbläsern, Streichern, Bass und Perkussion. Ende des 19. Jahrhunderts löste die Charanga das Orchester ab; in dem kleineren kammermusikartigen Ensemble 
wurden die Klarinetten durch Flöten und die Blechbläser durch eine Tres ${ }^{1}$ oder Harfe ersetzt. Anfang des 20. Jahrhunderts komplettierte das Klavier das zu Charanga Francesa umbenannte, bis heute übliche Ensemble, das jetzt aus Flöten, Streichern, Bass, Klavier und Perkussion (Güiro und Timbales oder Pailas) bestand (vgl. Giro 2004: 243f.).

Ein musikalisches Hauptcharakteristikum des Danzón ist die Verwendung des Cinquillo Cubano, die am frühesten belegte Clave in der kubanischen und karibischen Musik. Sie besteht aus der Formzahl 16 und der Impulszahl 9, notiert in Primer Tiempo [lollollololololo]. ${ }^{2}$ Ein weiteres Merkmal ist die Rondo-Form mit der Basisstruktur A-B-A-C-A. Der wiederkehrende Teil A ist die so genannte Introducción (dt. »Einführung«, »Einleitung«). Die anderen Formteile werden nach der Gruppe der Melodieinstrumente benannt, beispielsweise als Parte de Flauta (Flötenteil) und Parte Violín (Streicherteil). Eine bedeutende Formerweiterung war die Hinzufügung eines Montuno-Teils am Ende des Danzón (»Danzón con Montuno«): Der Montuno wurde in den 1910er Jahren vom bereits stärker an Popularität gewinnenden Son übernommen und diente zur Auflockerung der generell strengen Form, da er den Instrumentalisten die Möglichkeit zu Improvisationen bot. Als letztes musikalisches Charakteristikum sei erwähnt, dass seit Anfang des 20. Jahrhunderts häufig Melodien von bekannten Musikstücken (beispielsweise Themen aus Opern oder Operetten, Boleros, Chansons und Ragtimes) in die Danzón-Kompositionen eingebunden werden (vgl. Giro 2004: 245 und Sublette 2004: 309).

Das Stück »El Cadete Constitucional« wurde von Jacobo González Rubalcaba (1895-1960), Komponist, Dirigent und Musiker sowie Großvater von Gonzalo, in den 1920er Jahren komponiert. Für die Analyse wird die Aufnahme der Charanga Típica Cubana aus dem Jahr 1986 verwendet. Da die Zielsetzung dieser Aufnahme eine historisch möglichst korrekte Interpretation war, wird diese Version in den folgenden Analysen als »Original« be-

1 Die Tres ist ein von der Gitarre abstammendes, in Kuba entwickeltes Saiteninstrument mit drei üblicherweise doppelsaitigen Chören.

2 Für die Rhythmusanalysen wird die Impaktnotation verwendet: Ein Impakt wird als vertikaler Strich [I] notiert, ein Nonimpakt (leerer Puls, leerer Zählwert) als Punkt [o]. Die eckigen Klammern dienen der Abgrenzung von der Umgebung. Jede Clave hat eine gewisse Länge und besteht insofern aus einer gewissen Anzahl von Pulsen, was sich durch die Formzahl ausdrückt. Die Anzahl der Impakte innerhalb der Figur wird durch die Impulszahl benannt. Die besonders beliebten Claves mit der Formzahl 16 werden im musikalischen Gebrauch häufig in zwei Hälften, die so genannten Compases, unterteilt: Wird die Clave in ihrer ursprünglichen Form gespielt, erklingt sie in Primer Tiempo; werden die Compases vertauscht - also bei der Formzahl 16 die ersten acht Werte nach hinten gestellt -, wird sie in Segundo Tiempo gespielt (Hendler 2007: 17f. und 65). 
zeichnet. Musikalisch gesehen handelt es sich um einen Danzón con Montuno mit dem Cinquillo Cubano in Primer Tiempo als rhythmische Basis. Die Tonart ist F-Dur. Als spezielles Element wird im Teil C die Melodie von John Philip Sousas bekanntem Militärmarsch »The Stars And Stripes Forever « (1897) verwendet.

Von Gonzalo Rubalcaba sind zwei Aufnahmen dieses Danzón vorhanden: eine Trio-Version mit zusätzlicher Perkussion, aufgenommen im Jahr 2000 auf der CD Supernova ${ }^{3}$ und eine Solo-Version am Klavier, aufgenommen im Jahr 2010 auf der DVD Solos: The Jazz Sessions. Aus beiden Interpretationen werden in chronologischer Abfolge der Formteile die wesentlichen Analyseergebnisse, teilweise im Vergleich zum Original, dargestellt.

\begin{tabular}{|c|c|c|c|c|c|c|c|c|}
\hline Original & $A$ & B & \multicolumn{2}{|c|}{$A$} & $C$ & A & D & $E$ \\
\hline Teil & $\begin{array}{l}\text { Intro- } \\
\text { ducción }\end{array}$ & $\begin{array}{c}\text { Parte de } \\
\text { Flauta }\end{array}$ & \multicolumn{2}{|c|}{$\begin{array}{l}\text { Intro- } \\
\text { ducción }\end{array}$} & $\begin{array}{c}\text { Parte de } \\
\text { Violín }\end{array}$ & $\begin{array}{c}\text { Intro- } \\
\text { ducción }\end{array}$ & Coda & Montuno \\
\hline Takte & 16 & 16 & \multicolumn{2}{|c|}{16} & 32 & 16 & 24 & 116 \\
\hline $\begin{array}{l}\text { Trio- } \\
\text { Version }\end{array}$ & A & B & A & $\begin{array}{l}\text { Über- } \\
\text { leitung }\end{array}$ & $C$ & A & D & $E$ \\
\hline Takte & 16 & 16 & 8 & 4 & 32 & 8 & 24 & 122 \\
\hline $\begin{array}{l}\text { Solo- } \\
\text { Version }\end{array}$ & A & B & A & $\begin{array}{l}\text { Über- } \\
\text { leitung }\end{array}$ & C & A & & \\
\hline Takte & 16 & 16 & 8 & 4 & 32 & 4 & & \\
\hline
\end{tabular}

Tabelle 1: Vergleich der Formteile (Original, Trio- und Solo-Version)

Der Vergleich der formalen Abläufe zeigt, dass sich Rubalcaba in beiden Versionen in fast identischer Weise an diese charakteristische Rondo-Form des Danzón hält. Außerdem orientiert sich die Solo-Version an der zehn Jahre älteren Trio-Version.

\section{Introducción - Teil A}

Die 16-taktigen Introducciones setzen sich aus 8+8 Takten zusammen, wobei die jeweils ersten vier Takte das komponierte Thema enthalten, und die jeweils zweiten vier Takte unterschiedlich gespielt und daher als improvisiert bezeichnet werden. Die komponierten Takte werden in allen Versionen deutlich erkennbar realisiert:

3 Gonzalo Rubalcaba: Piano und Keyboards, Carlos Henríquez: Bass, Ignacio Berroa: Schlagzeug; Luís Quintero: Timbales und Güiro, Roberto Quintero: Conga. 


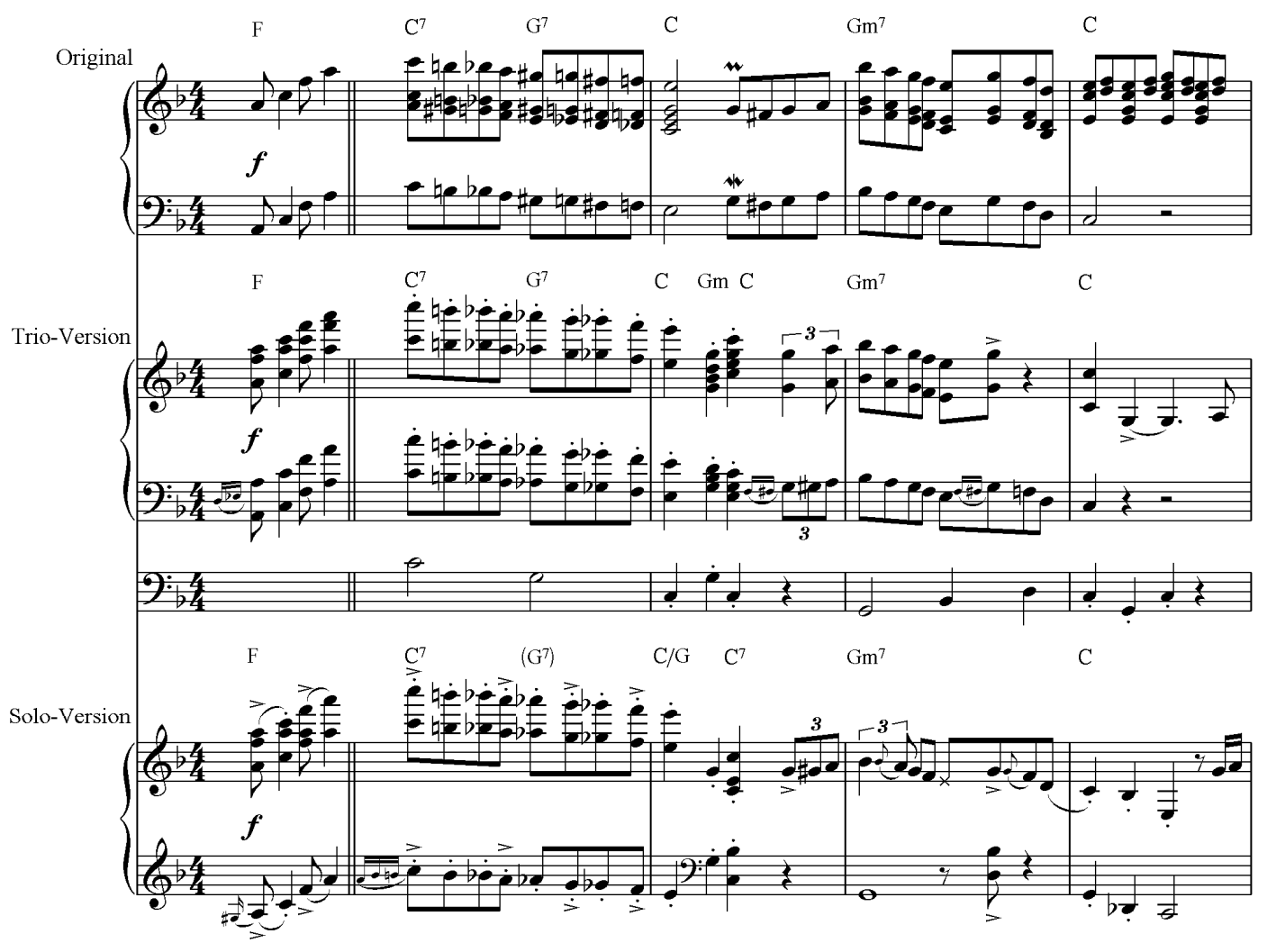

Notenbeispiel 1: Vergleich Introducción (Original, Trio- und Solo-Version), T. 1-4

Rubalcaba hält sich in beiden Versionen in melodischer und harmonischer Hinsicht an das Original und flechtet lediglich zahlreiche Verzierungen und Umspielungen ein. Abgesehen davon, dass der Cinquillo Cubano auch in der Trio-Version von den Perkussionsinstrumenten als rhythmische Basis erkennbar gespielt wird, kommt der Cinquillo [lollollo] außerdem auch im Melodierhythmus (hier: im Auftakt) vor.

Die jeweils improvisierten Taktteile bei Rubalcaba basieren auf demselben Harmonieschema, einer Erweiterung der Originalharmonien mit zusätzlichen II-V-Progressionen (Notenbeispiel 2). Basierend auf diesem harmonischen Gerüst, kreiert Rubalcaba jene 4-taktigen improvisierten Passagen, wobei er in diesen Teilen fern von der traditionellen kubanischen Stilistik und mit jazzidiomatischen Reharmonisationen agiert (Notenbeispiel 3). Außerdem hebt Rubalcaba diese eigenen Abschnitte hervor, indem er plötzlich die Dynamik reduziert und durch den starken dynamischen Kontrast die neue, eigene Melodie verdeutlicht. Dies ist in der Trio-Version besonders klar hörbar. 


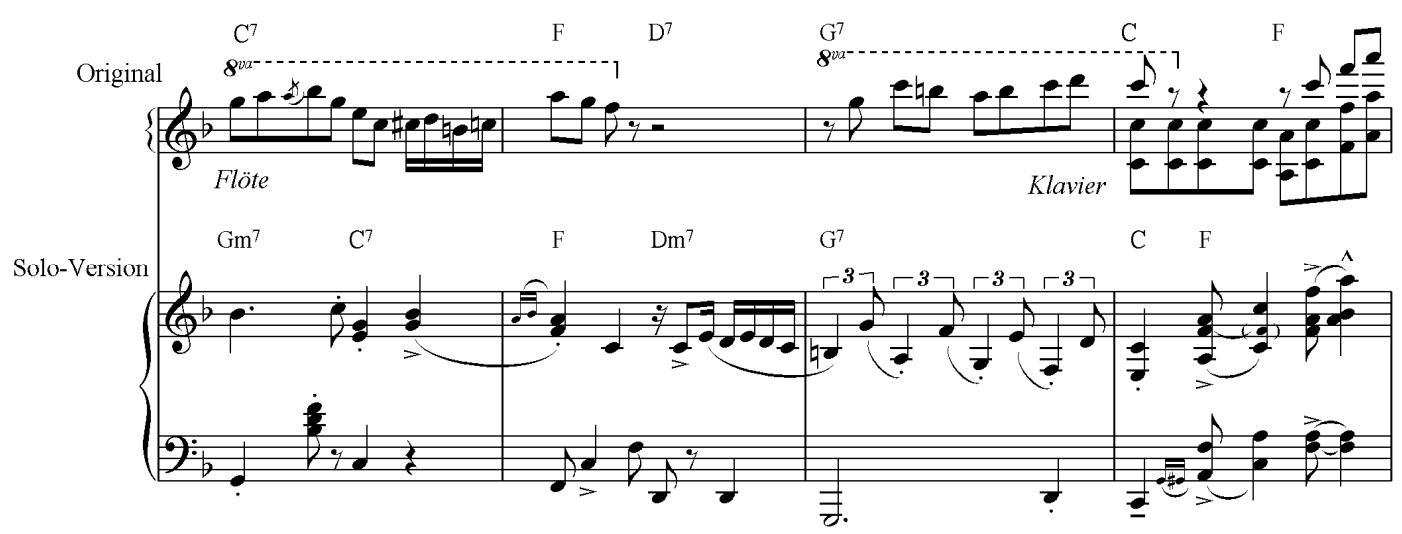

Notenbeispiel 2: Vergleich Introducción (Original und Solo-Version), T. 5-8

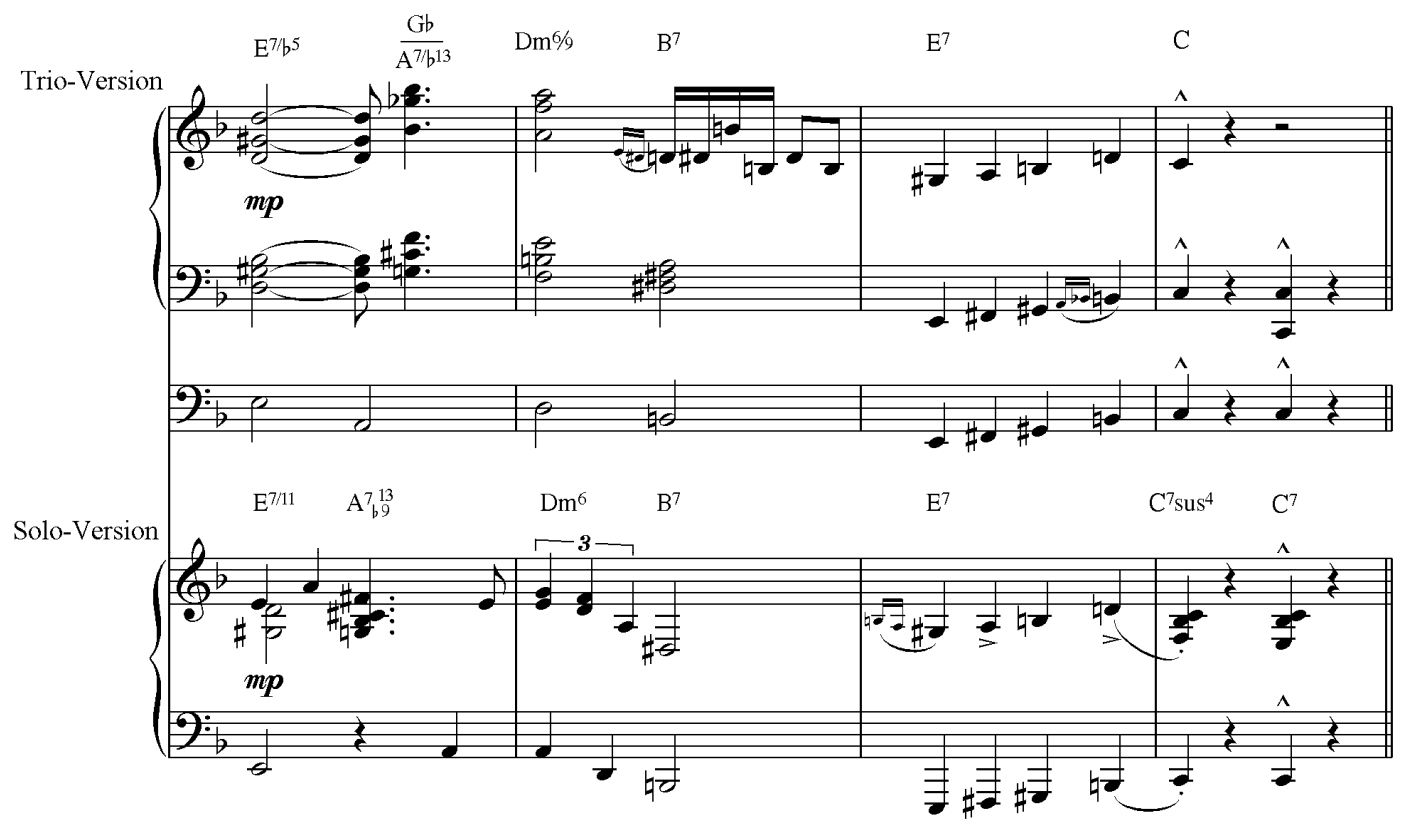

Notenbeispiel 3: Reharmonisation im zweiten A-Teil (Trio- und Solo-Version), T. 5-8

\section{Parte de Flauta - Teil B}

Auch in den B-Teilen beider Versionen behält Rubalcaba die melodischen und harmonischen Grundschemata bei. Abgesehen von melodischen und harmonischen Modifikationen ist jedoch eine Veränderung auffallend: Im Gegensatz zum Original, in dem dieser Teil recht laut und vom ganzen Ensemble (mit der Flöte als Hauptmelodieinstrument) gespielt wird, besticht Rubalcabas B-Teil in beiden Versionen durch einen sehr feinen und delikaten Charakter: 

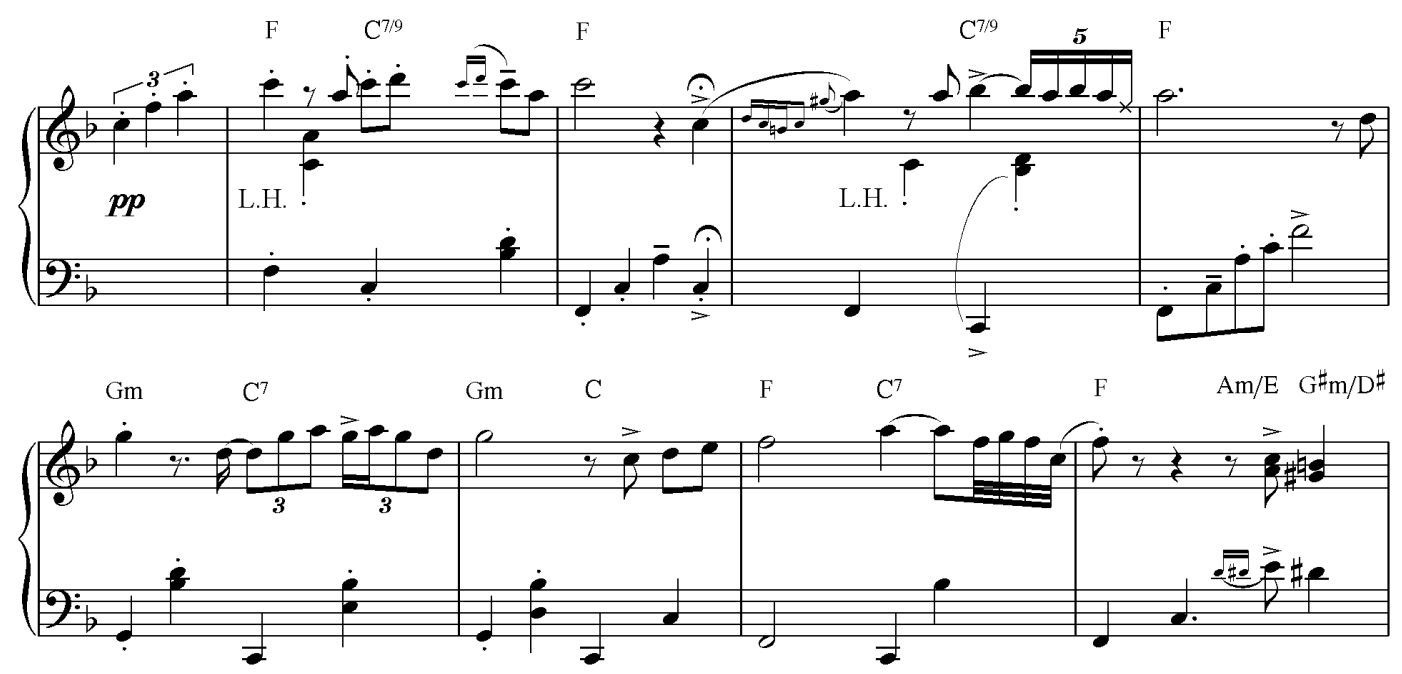

Notenbeispiel 4: Teil B der Solo-Version, T. 1-8

In der Trio-Version fällt in Takt 4 außerdem eine besondere Wendung auf, bei der Rubalcaba die Phrase anstatt mit dem üblicherweise gespielten $a^{2}$ mit einem $b^{2}$ beendet und dadurch eine unerwartete Reibung mit dem Grundton $f$ erzeugt:
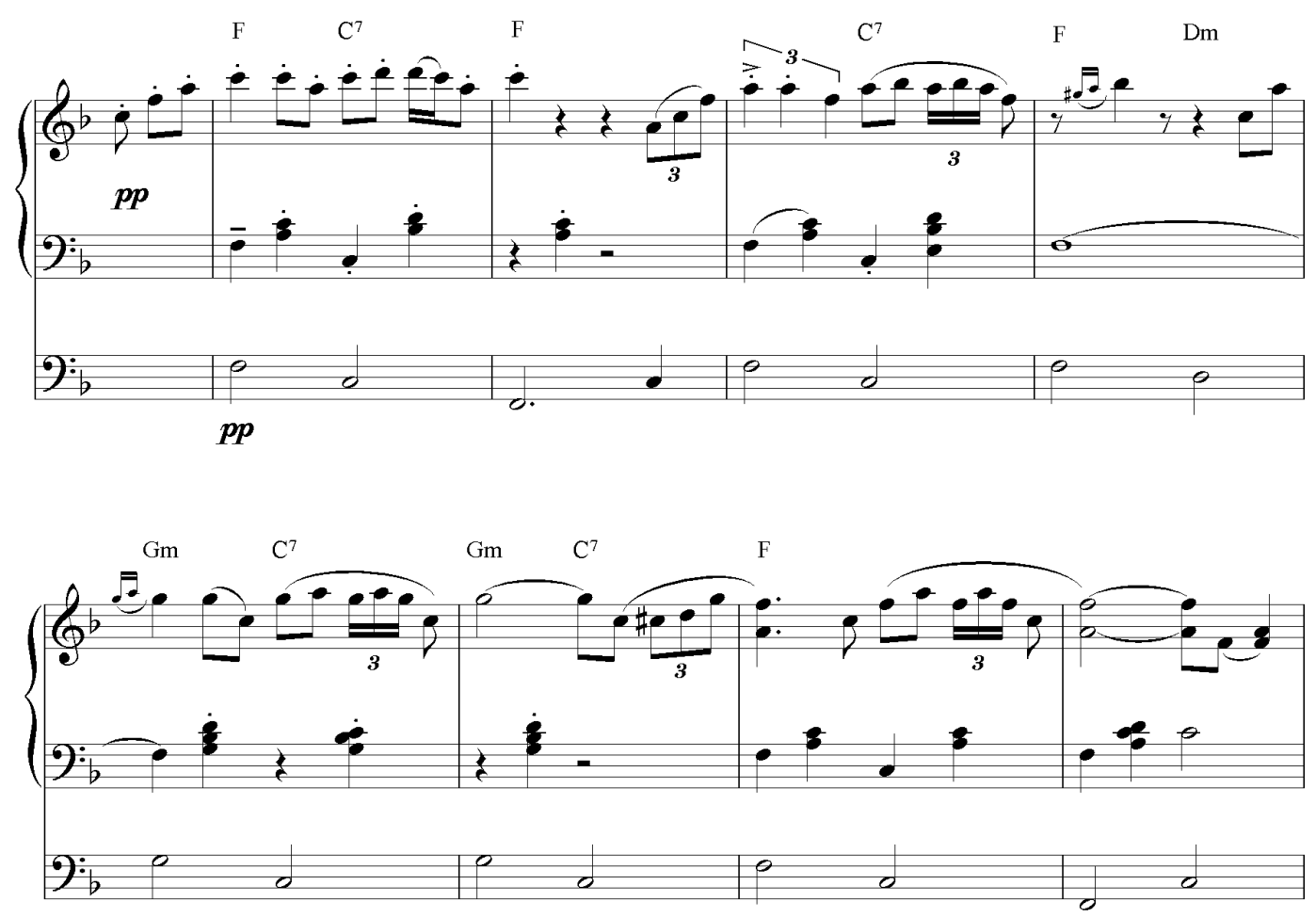

Notenbeispiel 5: Teil B der Trio-Version, T. 1-8 


\section{Parte de Violín - Teil C}

Die bekannte Melodie von »The Stars And Stripes Forever « wird - wie auch im Original - in Rubalcabas C-Teilen eingebaut. In der Trio-Version vom Bass legato mit dem Bogen eingespielt (mit leiser Klavier- und Perkussionsbegleitung) (Notenbeispiel 6), verändert Rubalcaba in der Solo-Version die Melodie durch einen Lagenwechsel: Er spielt die bekannte Melodie im Bereich der kleinen Oktave und die Begleitung reicht bis in die tiefsten Lagen des Klaviers (Notenbeispiel 7). Dadurch erhält diese Passage eine neue Klangfarbe.

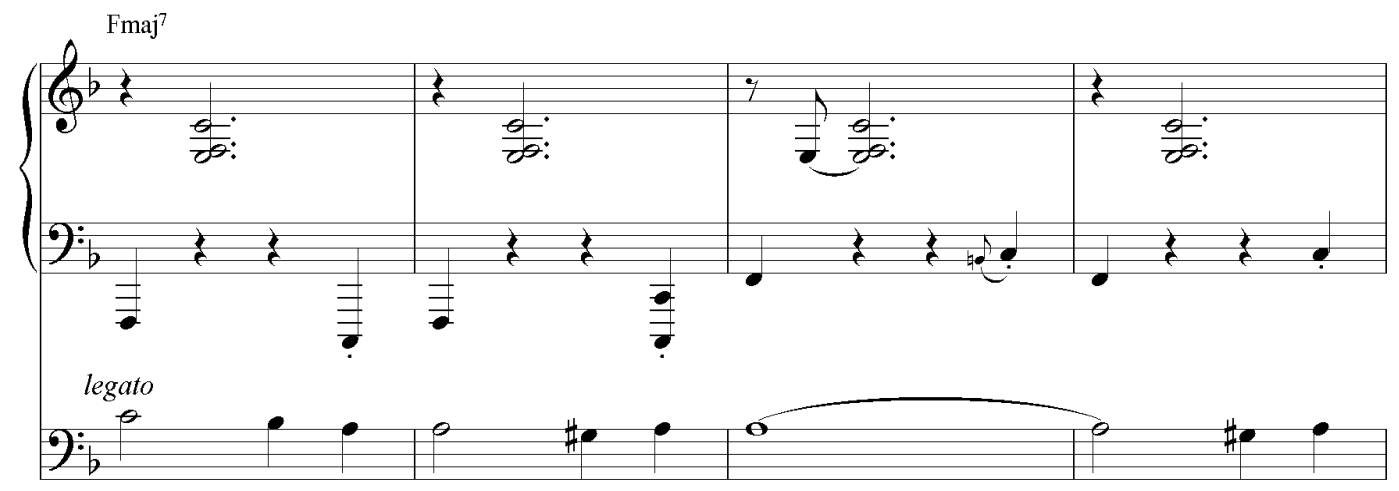

Notenbeispiel 6: Teil C der Trio-Version, T. 1-4
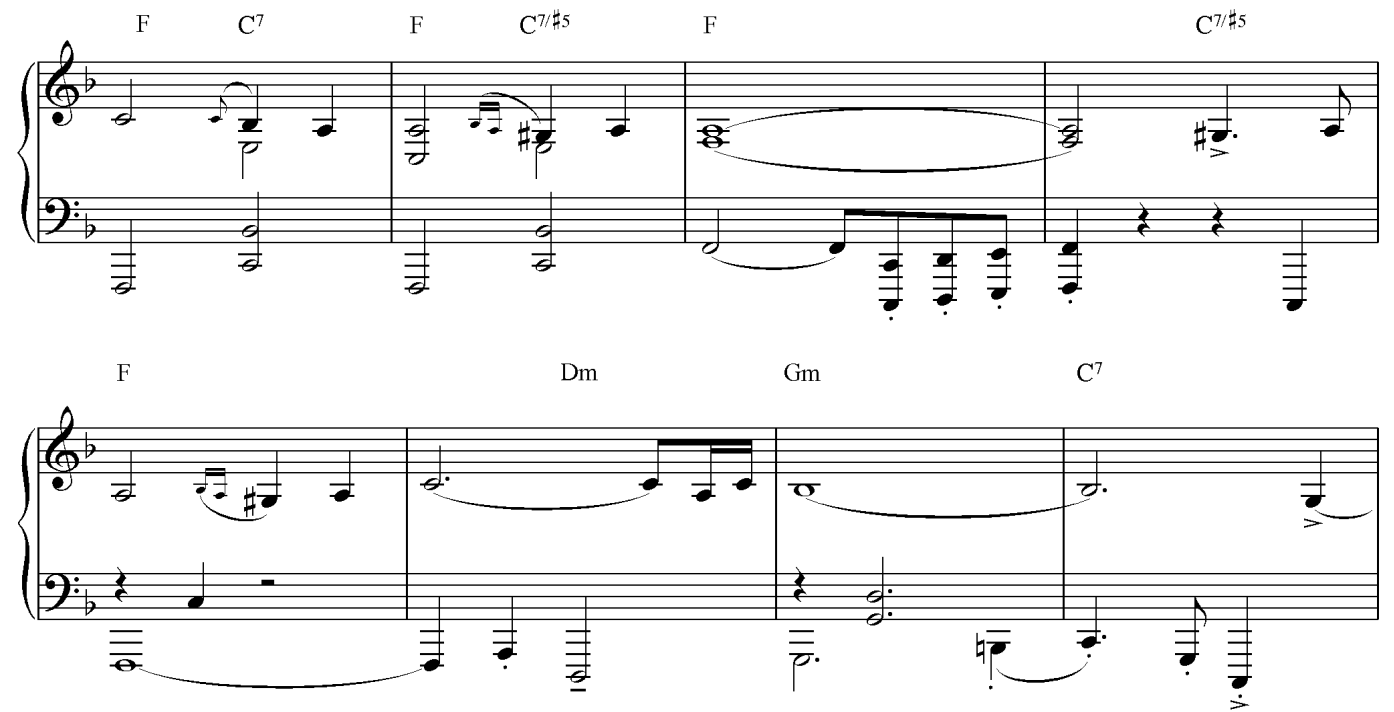

Notenbeispiel 7: Teil C der Solo-Version, T. 1-8 
In den Takten 17 bis 32 verändert Rubalcaba die Stilistik in beiden Interpretationen dahingehend, dass nach dem rhythmisch, melodisch und harmonisch eher einfachen und »korrekt« gespielten Marsch vermehrt dichte und jazzidiomatische Voicings eingesetzt werden:
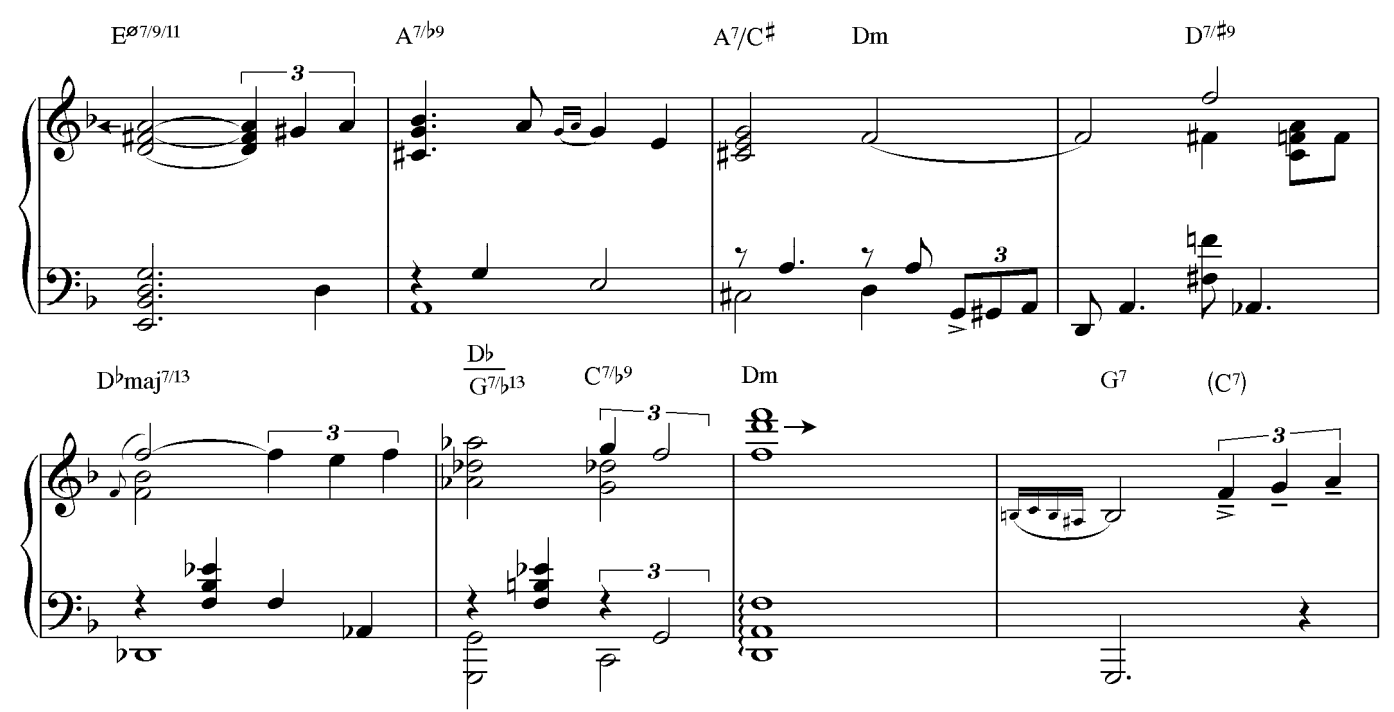

Notenbeispiel 8: Teil C der Solo-Version, T. 21-28

\section{Montuno}

Der Montuno wird im Original wie auch in Rubalcabas Trio-Version gespielt und basiert auf einer sich wiederholenden 2-taktigen Phrase über ein einfaches Harmonieschema $\left(I-\mid I-V^{7}\right)$. Dabei wird der Cinquillo Cubano in der Perkussion nicht mehr als rhythmische Grundlage verwendet. Dieses repetitive Pattern bildet die Basis für die Instrumentalimprovisationen:

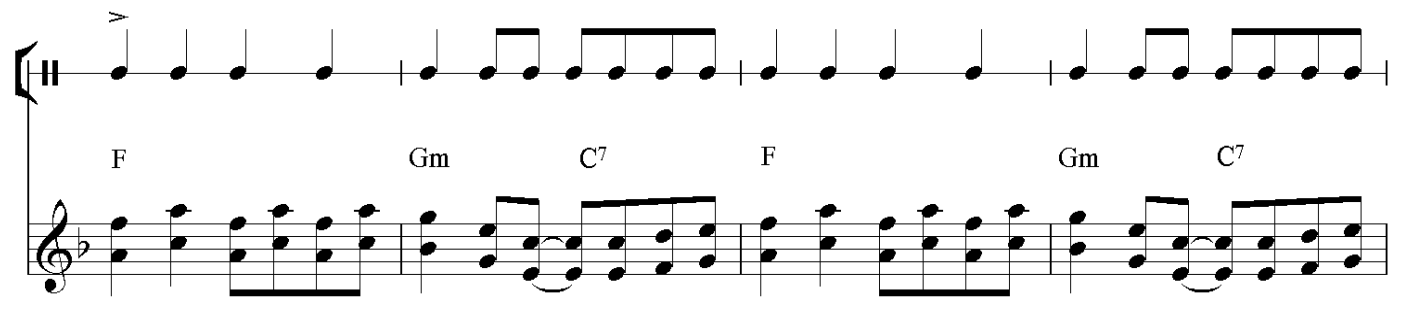

Notenbeispiel 9: Montuno-Pattern des Originals

Zunächst übernimmt Rubalcaba dieses Pattern in fast identischer Weise, mit der erweiterten Stufenfolge $\mathrm{I}-\mathrm{VI}-\mathrm{II}-\mathrm{V}^{7}$ : 


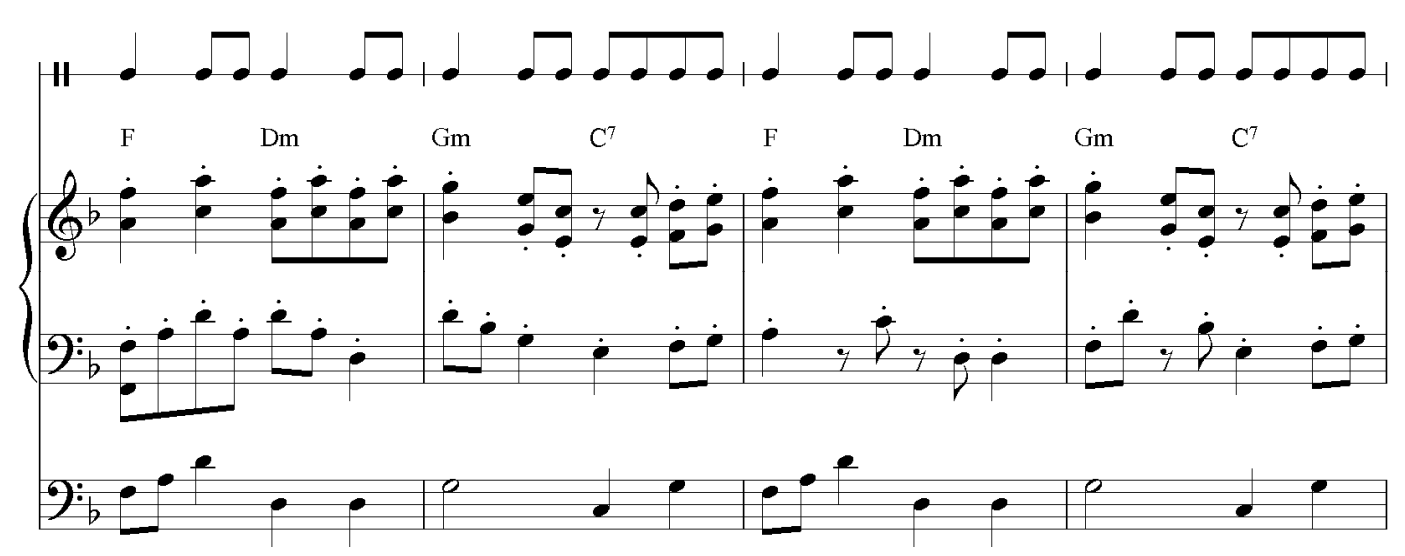

Notenbeispiel 10: Montuno-Pattern der Trio-Version

Nach mehreren Wiederholungen wechselt Rubalcaba zu einer neuen Figur als Improvisationsbasis: Er konstruiert eine eigene Clave mit der Formzahl 16 und zwei Compases (8+8 Grundpulse).

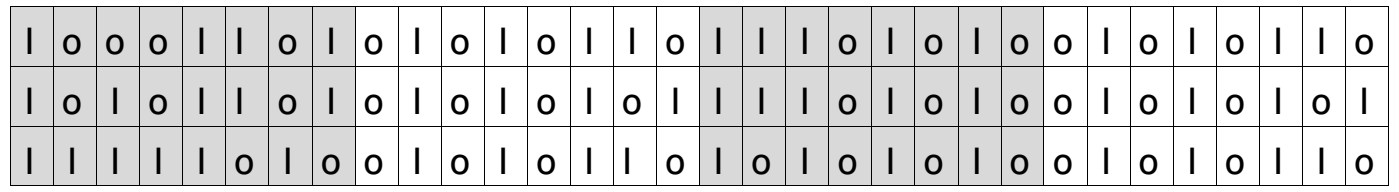

Tabelle 2: Montuno-Clave der Trio-Version

Obwohl diese Clave nicht konstant bleibt und exakt gleich wiederholt wird, ist doch ein fixiertes Grundkonzept erkennbar: Die Impaktanordnungen kehren - wenn auch nicht regelmäßig - wieder, und Puls eins beginnt immer mit einem Impakt, Puls neuen hingegen ohne einen Impakt (leerer Puls). Generell tendieren die ersten Compases (in grau) eher zu Impakten auf eins, drei, fünf und sieben, die zweiten Compases (in weiß) eher zu Impakten auf zwei, vier, sechs und acht. Die Impaktverschiebungen innerhalb dieser Claves in Kombination mit den unterschiedlichen Akzentuierungen und den teilweise etwas spät einsetzenden Impakten (Pfeil nach rechts, Notenbeispiel 11) erzeugen eine besondere rhythmische Spannung sowie eine gewisse rhythmische Irritation. Übertragen auf den 4/4-Takt sieht die Clave folgendermaßen aus: 

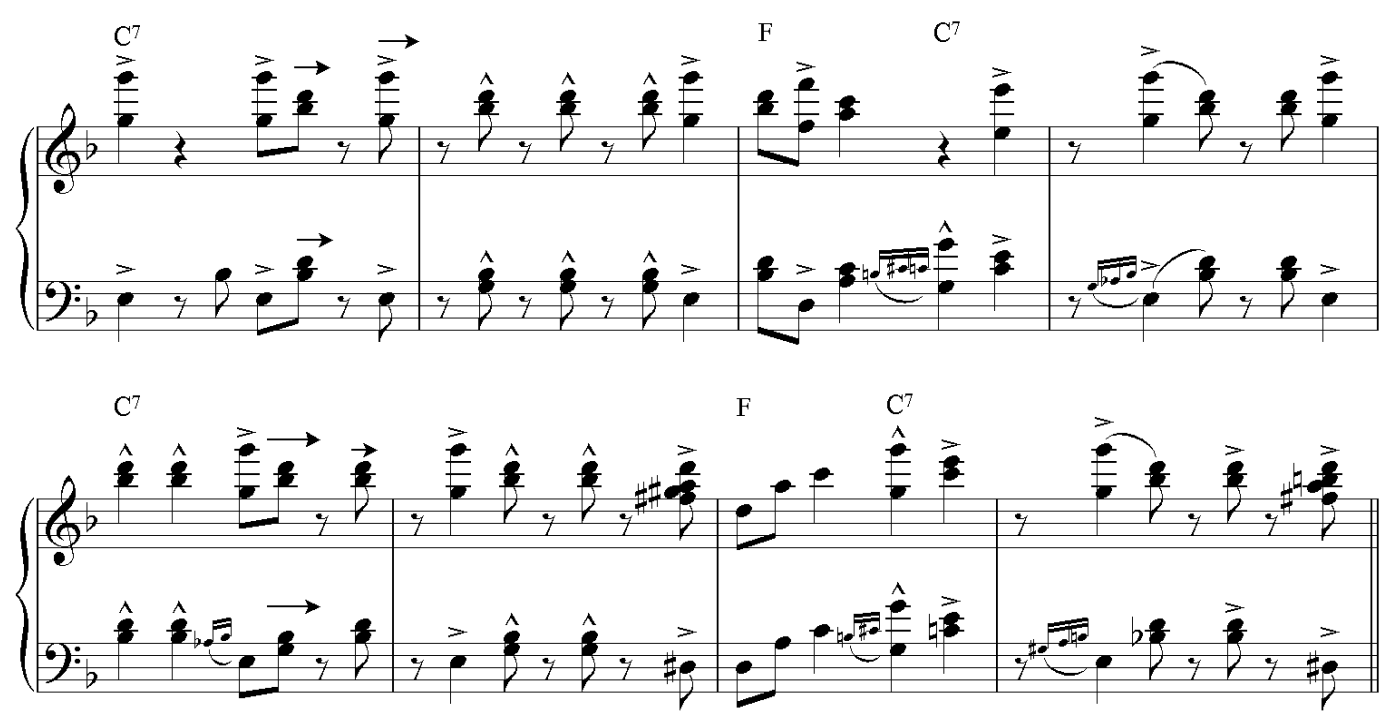

Notenbeispiel 11: Montuno-Clave der Trio-Version

\section{Schlussbemerkungen}

Die analytischen Betrachtungen der beiden Interpretationen von »El Cadete Constitucional« zeigen, dass Rubalcaba eine Vielzahl traditioneller Hauptcharakteristika des Danzón beibehält: Er übernimmt den Rondo-Formablauf, die Melodiestrukturen sowie die harmonischen Grundschemata, und auch die ursprünglich verwendete Clave wird in der Trio-Version als rhythmische Basis verwendet. Dennoch gelingt es Rubalcaba, mit den folgenden wesentlichen Gestaltungsmitteln neuartige Interpretationen des Danzón zu kreieren: Die Originalharmonik wird durch Reharmonisationen erweitert. Auch die Melodien werden variiert, wobei Rubalcaba sehr oft mit kleinen Umspielungen, rhythmischen Verschiebungen und unvorhergesehenen Wendungen arbeitet. Insgesamt gehören musikalisch unerwartete Wendungen und Kontraste (beispielsweise in den Bereichen Dynamik, Artikulation, Instrumentierung, Lagenwechsel, Stilistik und Charakter) zu den häufig angewandten Gestaltungsmitteln. Sind Harmonik und Melodik der Jazzidiomatik zuzurechnen, so bezieht sich Rubalcaba in der Rhythmik verstärkt auf die kubanische Tradition, indem er das Clave-Grundkonzept als Ausgangspunkt für rhythmische Umgestaltungen und Eigenkreationen verwendet, oft mit dem Ergebnis rhythmischer Komplexität und auch Irritation. Rubalcaba verwendet in den ausgewählten Interpretationen traditionelle kubanische Merkmale und verarbeitet sie in beachtenswerter Weise weiter, was in Kombination mit Elementen aus dem Jazz seinen Personalstil auszeichnet. 


\title{
Literatur
}

Dauer, Alfons Michael (1988). »Derler 1. Ein System zur Klassifikation von Rhythmen. Musiktheoretische und musikhistorische Aspekte. «In: Jazzforschung / Jazz Research 20. Graz: Akademische Druck- u. Verlagsanstalt, S. 117-154.

Díaz Ayala, Cristóbal (2011). Influencias recíprocas entre el jazz y la música caribeña. Unveröffentlichter Vortrag anlässlich El jazz desde la perspectiva caribeña: IV Congreso Internacional Música Identidad y Cultura en el Caribe. Santiago de los Caballeros (Dominikanische Republik), 15. bis 17. April.

Giro, Radamés (2004). „Kubanische Musik erobert die Welt: danzón, mambo und cha-cha-cha. « In: Alles in meinem Dasein ist Musik... Kubanische Musik von Rumba bis Techno. Hg. v. Torsten Esser u. Patrick Frölicher. Frankfurt/M.: Vervuert, S. 243-257.

Giro, Radamés (2007). Diccionario enciclopédico de la música en Cuba. Tomo 2. La Habana: Editorial Letras Cubanas.

Gómez Sotolongo, Antonio (2011). Apuntes para un estudio del jazz en Cuba o las consecuencias del lleva y trae entre el jazz y la música cubana. Unveröffentlichter Vortrag anlässlich El jazz desde la perspectiva caribeña: IV Congreso Internacional Música Identidad y Cultura en el Caribe. Santiago de los Caballeros (Dominikanische Republik), 15. bis 17. April.

Hendler, Maximilian (2007). Clave del Son. Die Rhythmusformeln in der Musik der Karibik (= Musikwissenschaft 12). Münster: Lit-Verlag.

Sublette, Ned (2004). Cuba and Its Music. From the First Drums to the Mambo. Chicago: Chicago Review Press.

\section{Diskografie}

Centre de Développement et de Recherche des Archives de la Musique Cubaine (1999). »El Cadete Constitucional.«Auf: Rétrospective Officielle Des Musiques Cubaines, Vol. 4, Frémeaux Et Associés FA 176.

Rubalcaba, Gonzalo (2001). »El Cadete Constitucional.«Auf: Supernova, Blue Note 5-31172-2.

Rubalcaba, Gonzalo (2010). »Improvisation \#1 (El Cadete Constitucional).« Auf: Solos. The Jazz Series, MVD Visual MVD 4964D.

\begin{abstract}
This paper studies two contemporary jazz versions of the traditional Cuban danzón »El Cadete Constitucional« by Gonzalo Rubalcaba. An introduction into the main characteristics of the danzón is given. Based on transcribed examples, it is shown how jazz pianist and composer Rubalcaba transforms traditional Cuban elements of the danzón and uses them in his contemporary jazz performances.
\end{abstract}

